\title{
La prévention des facteurs de risque cardiovasculaire chez la personne âgée
}

\section{Prevention of cardiovascular risk factors in elderly}

\author{
T. Vogel \\ C) Springer-Verlag France 2013
}

« If a man will begin with certainties, he shall end in doubts; but if he will be content to begin with doubts, he shall end in certainties $»$.

Sir Francis Bacon, The Advancement of Learning, 1605

Pour l'INSEE, selon l'hypothèse d'un scénario central (ou tendantiel), la France devrait compter en 205070 millions d'habitants dont 22,3 millions seraient âgés de plus de 60 ans contre 12,6 millions en 2005, soit une hausse de $80 \%$ en 45 ans [1].

Les décès d'origine cardiovasculaire augmentent avec l'avancée en âge, devenant la principale cause de décès après 85 ans, devant les décès liés au cancer (pour les 85-94 ans : $35 \%$ de décès d'origine cardiovasculaire et $18 \%$ de décès liés au cancer; pour les 95 ans et plus : $37 \%$ de décès d'origine cardiovasculaire et $8 \%$ de décès liés au cancer).

Dans ce contexte, la notion de prévention chez le senior, tout particulièrement cardiovasculaire, prend tout son sens, notamment chez les sujets très âgés.

C'est la thématique que nous abordons dans ce numéro des Cahiers de l'Année Gérontologique.

Concernant les facteurs de risque cardiovasculaire, nous avons choisi de développer la prise en charge du diabète et de l'hypertension artérielle chez le sujet âgé et surtout très âgé. De nouvelles recommandations ont en effet été publiées pour ces deux facteurs de risque, tant au niveau national qu'européen. Volontairement, nous n'aborderons pas la problématique des dyslipémies chez le sujet âgé de plus de 80 ans, faute de données récentes sur la question. Depuis l'étude PROSPER de 2002, aucun essai randomisé contrôlé incluant exclusivement des sujets âgés et évaluant des critères de jugement de morbimortalité n'a été publié [2]... Des études avec des critères de jugement intermédiaires [3], des

\section{T. Vogel $(\square)$}

Professeur des Universités, praticien hospitalier, chef de service du secteur SSRG, pôle de gériatrie,

hôpitaux universitaires de Strasbourg

e-mail : thomas.vogel@chru-strasbourg.fr méta-analyses $[4,5]$ et des recommandations ont été publiées [6] mais sans démonstration d'une diminution de la mortalité après 80 ans [7-9]. Nous voilà donc plongés depuis plus de dix années dans l'incertitude thérapeutique quant au bénéfice sur la mortalité des statines chez les plus de 80 ans. Pour illustrer cette incertitude, je projette aux étudiants une photographie dans le brouillard de notre belle cathédrale de Strasbourg (Fig. 1) Nonobstant ces incertitudes, les statines sont pourtant prescrites à large échelle chez les sujets de plus

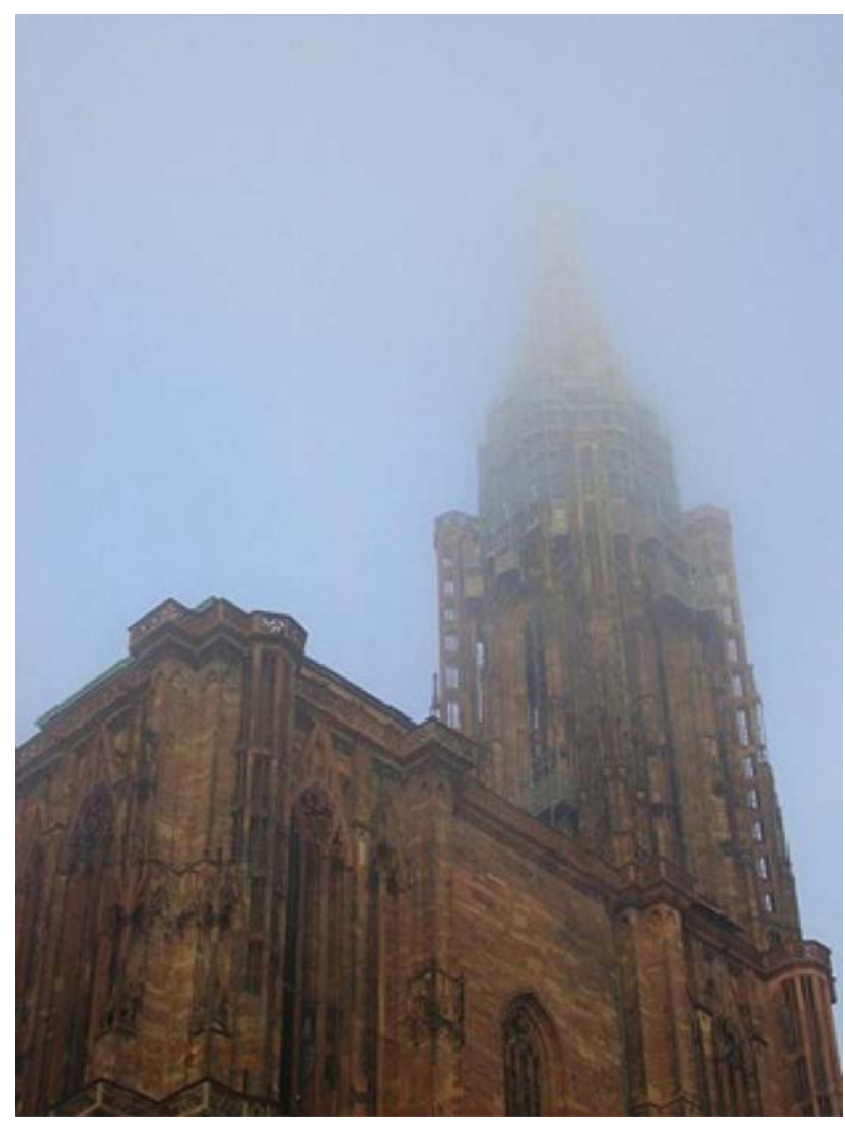

Fig. 1 La cathédrale de Strasbourg dans le brouillard à l'image du bénéfice sur la mortalité du traitement par statines après 80 ans... 
de 80 ans, appuyées par un lobbying marketing puissant et par la notion théorique de leurs effets pléiotropes qui va au-delà de la simple baisse du cholestérol [10]. N'oublions pas les effets secondaires des statines et notamment leur toxicité musculaire chez le sujet âgé...

Nous nous intéressons ensuite au déclin cognitif et à sa composante vasculaire à partir de deux études cliniques, l'étude PARTAGE (sujets $>80$ ans institutionnalisés) et l'étude ADELAHYDE (sujets hypertendus âgés de 60 à 85 ans avec plainte mnésique) menées au CHU de Nancy.

Nous faisons également une mise au point concernant le traitement antithrombotique dans la fibrillation atriale qui avec l'arrivée des nouveaux anticoagulants (NACO) connaît un tournant évolutif majeur. Là encore, les NACO n'ont pas été évalués à large échelle chez les sujets de plus de 80 ans, et à ce jour il n'y a toujours pas d'antidotes disponibles. Peut-on (doit-on ?) prescrire à des personnes âgées des molécules qui n'ont pas été évaluées après 80 ans à large échelle dans les études de phase III? Primum non nocere...

Nous illustrons la problématique du traitement antithrombotique dans la fibrillation atriale par une étude originale effectuée dans 11 EHPAD de Strasbourg.

Nous terminons ce numéro par une mise au point concernant les bénéfices pour la santé chez le senior d'une activité physique régulière en endurance. Nous insisterons sur les bénéfices apportés par l'activité physique en oncologie, en cognition et en physiologie cardiorespiratoire. Enfin, nous parlerons de la Consultation de l'Aptitude Physique pour la Santé (CAPS) que nous avons mise en place au pôle de gériatrie des hôpitaux universitaires de Strasbourg, en rappelant les résultats de l'étude de validation du programme spécifique de réentraînement qui est proposé (le Programme d'Entraînement Personnalisé sur Cycle ou PEP'C)

Remerciements Je tiens à remercier chaleureusement l'ensemble des auteurs qui ont participé à la rédaction de ces articles, tout particulièrement le Pr Blicklé, le Dr Watfa et Mme le Dr Suna-Enache.

\section{Références}

1. INSEE. consulté en août 2013 : www.insee.fr

2. Shepherd J, Blauw GJ, Murphy MB, et al (2002) PROspective Study of Pravastatin in the Elderly at Risk. Pravastatin in elderly individuals at risk of vascular disease (PROSPER): a randomised controlled trial. Lancet 360:1623-30

3. Deedwania P, Stone PH, Bairey Merz CN, et al (2007) Effects of intensive versus moderate lipid-lowering therapy on myocardial ischemia in older patients with coronary heart disease: results of the Study Assessing Goals in the Elderly (SAGE). Circulation 115:700-7

4. Afilalo J, Duque G, Steele R, et al (2008) Statins for secondary prevention in elderly patients: a hierarchical bayesian metaanalysis. J Am Coll Cardiol 51:37-45

5. Gränsbo K, Melander O, Wallentin L, et al (2010) Cardiovascular and cancer mortality in very elderly post-myocardial infarction patients receiving statin treatment. J Am Coll Cardiol 55:1362-9

6. Catapano AL, Reiner Z, De Backer G, et al (2011) ESC/EAS Guidelines for the management of dyslipidaemias The Task Force for the management of dyslipidaemias of the European Society of Cardiology (ESC) and the European Atherosclerosis Society (EAS). Atherosclerosis 217:3-46

7. Petersen LK, Christensen K, Kragstrup J (2010) Lipid-lowering treatment to the end? A review of observational studies and RCTs on cholesterol and mortality in 80 +- year olds. Age Ageing 39:674-80

8. Savarese G, Gotto AM Jr, Paolillo S, et al (2013) Benefits Of Statins In Elderly Subjects Without Established Cardiovascular Disease. A Meta-Analysis. J Am Coll Cardiol 13:S0735-1097

9. Gnjidic D, Le Couteur DG, Blyth FM, et al (2013) Statin use and clinical outcomes in older men: a prospective population-based study. BMJ Open 3:pii:e002333

10. Boccardi V, Barbieri M, Rizzo MR, et al (2013) A new pleiotropic effect of statins in elderly: modulation of telomerase activity. FASEB J [Epub ahead of print] 